\title{
Facile and Time-Efficient Carboxylic Acid Functionalization of PIM-1: Effect on Molecular Packing and Gas Separation Performance
}

\section{Supporting Information}

Katherine Mizrahi Rodriguez ${ }^{1}$, Albert X. Wu ${ }^{2}$, Qihui Qian², Gang Han², Sharon Lin $^{2}$, Francesco M. Benedetti ${ }^{2}$, Hyunhee Lee ${ }^{2}$, Won Seok $\mathrm{Chi}^{3}$, Cara M. Doherty ${ }^{4}$, and Zachary P. Smith ${ }^{2, *}$

${ }^{1}$ Department of Materials Science and Engineering, Massachusetts Institute of Technology, Cambridge, Massachusetts 02139, United States

${ }^{2}$ Department of Chemical Engineering, Massachusetts Institute of Technology, Cambridge, Massachusetts 02139, United States

${ }^{3}$ School of Polymer Science and Engineering, Chonnam National University, 77 Yongbong-ro Buk-gu, Gwangju 61186, Korea

${ }^{4}$ The Commonwealth Scientific and Industrial Research Organization (CSIRO), Private Bag 10, Clayton South, Victoria 3169, Australia 


\section{S1. Solvent solubility of PIM-COOH}

PIM-COOH solubility: The deprotonated PIM-COOH powder was difficult to dissolve in DMF, DMSO, and THF, requiring rapid stirring rates and heating to achieve full dissolution. As a result, the post-reaction procedure was modified to include washing with slightly acidified DI water to retain the protonated carboxylic acid. After washing, it was found that, due to its polarity, PIM$\mathrm{COOH}$ is highly soluble in strong polar aprotic solvents, including DMF and DMSO, and it is also soluble in less polar solvents, including THF. PIM-1 is soluble in slightly polar solvents including $\mathrm{CHCl}_{3}, \mathrm{DCM}$, and THF, but it is insoluble in the strong polar aprotic solvents, including DMF and DMSO. Such solubility differences arise from the differences in chemical composition of the polymers.

\section{S2. FT-IR}

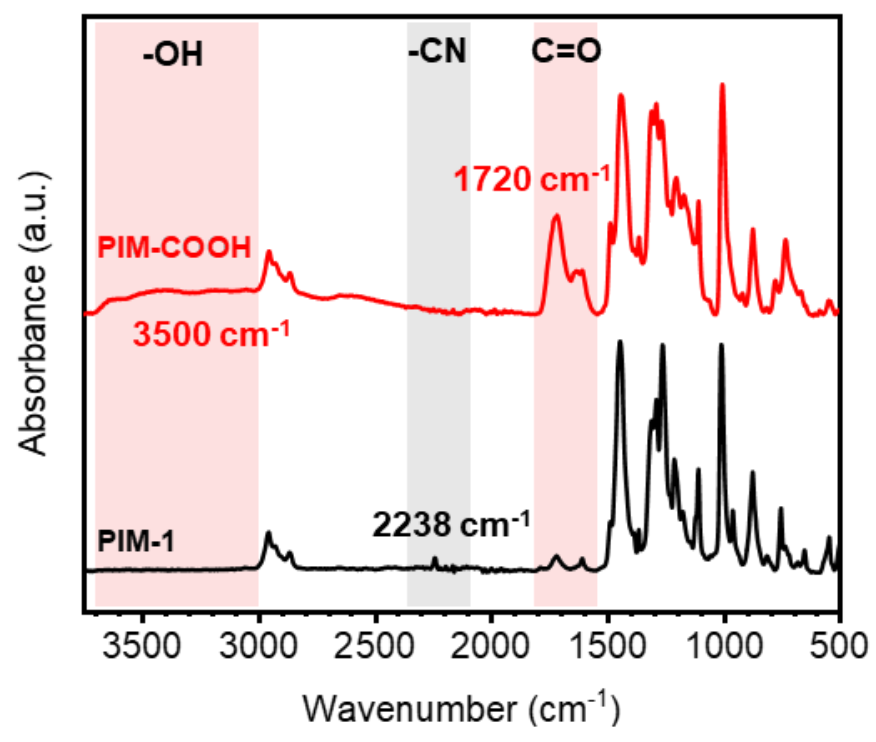

Figure S1. FT-IR spectra of a PIM-1 and a PIM-COOH film. Bands associated with the carboxylic acid and nitrile moieties are highlighted in red and gray, respectively. 


\section{S3. Elemental Analysis}

Table S1. XPS analysis of PIM-1 and PIM-COOH film samples.

\begin{tabular}{|c|c|c|c|c|l|l|}
\hline \multirow{2}{*}{ Sample } & \multicolumn{2}{|c|}{ PIM-1 } & \multicolumn{2}{c|}{ PIM-COOH } & PIM-CONH \\
\cline { 3 - 7 } & & Exp. & Predicted & Exp. & Predicted & Predicted \\
\hline \multirow{3}{*}{$\begin{array}{c}\text { Atomic } \\
\%\end{array}$} & $\mathbf{C}$ & 82.0 & 82.9 & 79.5 & 78.4 & 78.4 \\
\cline { 3 - 7 } & $\mathbf{O}$ & 12.7 & 11.4 & 20.3 & 21.6 & 16.2 \\
\cline { 3 - 7 } & $\mathbf{N}$ & 5.3 & 5.7 & 0.2 & 0 & 5.4 \\
\hline
\end{tabular}

\section{S4. Permeation Measurements}

Table S2. Permeability, sorption, and diffusivity for PIM-1 and PIM-COOH films calculated using the time-lag method from permeation tests performed at $1 \mathrm{~atm}$ and $35^{\circ} \mathrm{C}$. Time-lag analysis was not performed for $\mathrm{H}_{2}$ due to uncertainties associated with fast permeation. Values are averages of at least three measurements, and error bars indicate standard deviations.

\begin{tabular}{|c|c|r|c|c|c|c|c|}
\hline \multicolumn{7}{|c|}{ Values calculated from time-lag method and $\boldsymbol{P}=\boldsymbol{D} \times \boldsymbol{S}$} \\
\hline \multirow{2}{*}{ Gas } & \multicolumn{2}{|c|}{ Permeability (barrer) } & \multicolumn{2}{|c|}{ Sorption $\left(\mathrm{cm}^{3}{ }_{\text {STP }} \mathrm{cm}^{-3}{ }_{\text {pol }} \mathrm{atm}^{-1}\right)$} & \multicolumn{1}{l|}{ Diffusivity $\left(10^{-7} \mathrm{~cm}^{-2} \mathrm{~s}^{-1}\right)$} \\
\cline { 2 - 8 } & PIM-1 & PIM-COOH & PIM-1 & PIM-COOH & PIM-1 & PIM-COOH \\
\hline $\mathbf{H}_{\mathbf{2}}$ & $1400 \pm 200$ & $270 \pm 40$ & - & - & - & - \\
\hline $\mathbf{N}_{\mathbf{2}}$ & $140 \pm 30$ & $9.0 \pm 2$ & $2.1 \pm 0.2$ & $0.9 \pm 0.3$ & $5.0 \pm 1$ & $0.8 \pm 0.3$ \\
\hline $\mathbf{O}_{\mathbf{2}}$ & $460 \pm 80$ & $44 \pm 9$ & $2.5 \pm 0.1$ & $1.3 \pm 0.2$ & $14 \pm 2$ & $2.6 \pm 0.6$ \\
\hline $\mathbf{C H}_{\mathbf{4}}$ & $220 \pm 50$ & $9.0 \pm 2$ & $8.1 \pm 0.4$ & $4.4 \pm 1$ & $2.1 \pm 0.5$ & $0.16 \pm 0.02$ \\
\hline $\mathbf{C O}_{\mathbf{2}}$ & $2800 \pm 500$ & $290 \pm 60$ & $30 \pm 1$ & $23 \pm 2$ & $7.0 \pm 1$ & $1.0 \pm 0.2$ \\
\hline
\end{tabular}


Table S3. Permeability, sorption, and diffusivity selectivities for PIM-1 and PIM-COOH films calculated using the time-lag method for permeation tests performed at 1 atm and $35^{\circ} \mathrm{C}$. Values displayed are averages of at least three measurements, and error bars indicate standard deviations.

\begin{tabular}{|c|c|c|c|c|c|c|}
\hline \multicolumn{7}{|c|}{ Values calculated from time-lag method and $P=D \times S$} \\
\hline \multirow{2}{*}{ Gas Pair } & \multicolumn{2}{|c|}{ Permselectivity $\left(\alpha_{P}\right)$} & \multicolumn{2}{|c|}{ Sorption selectivity $\left(\alpha_{S, \theta}\right)$} & \multicolumn{2}{|c|}{ Diffusivity selectivity $\left(\alpha_{D, \theta}\right)$} \\
\hline & PIM-1 & PIM-COOH & PIM-1 & PIM-COOH & PIM-1 & PIM-COOH \\
\hline $\mathrm{CO}_{2} / \mathrm{CH}_{4}$ & $13 \pm 0.7$ & $33 \pm 2$ & $3.8 \pm 0.1$ & $6.0 \pm 2$ & $3.4 \pm 0.3$ & $6.0 \pm 2$ \\
\hline $\mathrm{CO}_{2} / \mathrm{N}_{2}$ & $20 \pm 0.7$ & $32 \pm 2$ & $14 \pm 1$ & $26 \pm 2$ & $1.4 \pm 0.1$ & $1.3 \pm 0.3$ \\
\hline $\mathbf{H}_{2} / \mathbf{N}_{2}$ & $10 \pm 0.8$ & $31 \pm 1$ & - & - & - & - \\
\hline $\mathrm{O}_{2} / \mathbf{N}_{2}$ & $3.2 \pm 0.1$ & $5.0 \pm 0.1$ & $1.2 \pm 0.1$ & $1.5 \pm 0.1$ & $2.7 \pm 0.3$ & $3.5 \pm 0.6$ \\
\hline
\end{tabular}




\section{S5. Sorption Analysis}

Table S4. Dual-mode sorption (DMS) model parameters from sorption isotherms at $35^{\circ} \mathrm{C}$.

\begin{tabular}{|c|c|c|c|c|c|c|c|c|c|c|c|}
\hline \multirow{2}{*}{ Sample } & \multirow{2}{*}{\begin{tabular}{|c} 
Gas \\
Constrain \\
Pressure
\end{tabular}} & \multicolumn{2}{|c|}{$k_{D}^{\mathrm{a}, \mathrm{d}}$} & \multicolumn{2}{|c|}{$C_{H}^{\prime, \mathrm{a}, \mathrm{e}}$} & \multicolumn{2}{|c|}{$\boldsymbol{b}\left(\mathrm{atm}^{-1}\right)^{\mathbf{a}}$} & \multicolumn{2}{|c|}{$S^{\infty} \mathbf{b , d}$} & \multicolumn{2}{|c|}{$\frac{C_{H}^{\prime} \times b}{k_{D}}$} \\
\hline & & 10 atm & $40 \mathrm{~atm}$ & $10 \mathrm{~atm}$ & $40 \mathrm{~atm}$ & $10 \mathrm{~atm}$ & $40 \mathrm{~atm}$ & $10 \mathrm{~atm}$ & $40 \mathrm{atn}$ & $10 \mathrm{~atm}$ & $40 \mathrm{~atm}$ \\
\hline \multirow{3}{*}{ PIM-1 } & $\mathbf{N}_{2}$ & 0.233 & 0.182 & 44.1 & 49.8 & 0.045 & 0.039 & 2.21 & 2.14 & 8.51 & 10.8 \\
\hline & $\mathrm{CH}_{4}$ & 0.565 & 0.506 & 64.2 & 66.5 & 0.109 & 0.109 & 7.56 & 7.78 & 12.4 & 14.4 \\
\hline & $\mathrm{CO}_{2}$ & 2.67 & 3.04 & 82.5 & 73.4 & 0.515 & 0.658 & 45.2 & 51.36 & 15.9 & 15.9 \\
\hline \multirow{3}{*}{$\begin{array}{c}\text { PIM- } \\
\text { COOH }\end{array}$} & $\mathbf{N}_{2}$ & 0.216 & 0.197 & 24.8 & 27.5 & 0.049 & 0.044 & 1.43 & 1.41 & 5.64 & 6.12 \\
\hline & $\mathrm{CH}_{4}$ & 0.471 & 0.443 & 42.0 & 44.1 & 0.107 & 0.099 & 4.98 & 4.81 & 9.56 & 9.83 \\
\hline & $\mathrm{CO}_{2}$ & 1.85 & 1.84 & 63.3 & 63.6 & 0.420 & 0.410 & 28.5 & 28.0 & 14.4 & 14.2 \\
\hline
\end{tabular}

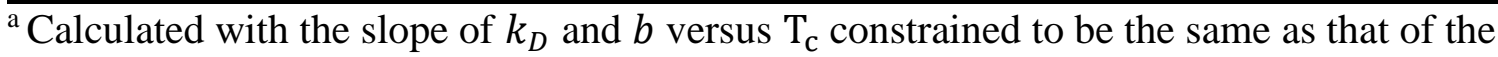
sorption coefficient against $\mathrm{T}_{\mathrm{c}}$ at $10 \mathrm{~atm}$ or 40 atm as described by Smith $e t$ al. ${ }^{1}$

${ }^{\mathrm{b}}$ Calculated using the dual-mode sorption model equation with unconstrained best-fit parameters.

${ }^{c}$ Pressure at which the slope of the sorption coefficient against $T_{c}$ was measured to constrain the analysis of DMS parameters.

${ }^{\mathrm{d}}$ Units of $\mathrm{cm}^{3}{ }_{\mathrm{STP}} \mathrm{cm}^{-3}{ }_{\text {pol }} \mathrm{atm}^{-1}$.

${ }^{\mathrm{e}}$ Units of $\mathrm{cm}^{3}{ }_{\text {STP }} \mathrm{cm}^{-3}{ }_{\text {pol }}$ 
Table S5. Sorption selectivity at 1 atm $\left(\alpha_{S, s e c}\right)$ and at infinite dilution $\left(\alpha_{S, s e c}^{\infty}\right)$, and diffusivity selectivity at $1 \mathrm{~atm}\left(\alpha_{D, s e c}\right)$ calculated using the secant method from sorption. Values are calculated using the best-fit dual-mode sorption parameters without constraints.

\begin{tabular}{|l|l|l|l|l|l|l|}
\hline \multirow{2}{*}{ Gas Pair } & \multicolumn{2}{|c|}{$\boldsymbol{\alpha}_{S, \text { sec }}$} & \multicolumn{2}{c|}{$\boldsymbol{\alpha}_{S, \text { sec }}^{\infty}$} & \multicolumn{2}{c|}{$\boldsymbol{\alpha}_{D, \text { sec }}$} \\
\cline { 2 - 7 } & \multicolumn{1}{|c|}{ PIM-1 } & PIM-COOH & PIM-1 & PIM-COOH & PIM-1 & PIM-COOH \\
\hline $\mathbf{C O}_{\mathbf{2}} / \mathbf{C H}_{4}$ & 3.7 & 4.0 & 6.0 & 4.9 & 3.4 & 8.1 \\
\hline $\mathbf{C O}_{\mathbf{2}} / \mathbf{N}_{\mathbf{2}}$ & 15 & 16 & 20 & 22 & 1.3 & 2.0 \\
\hline
\end{tabular}


A

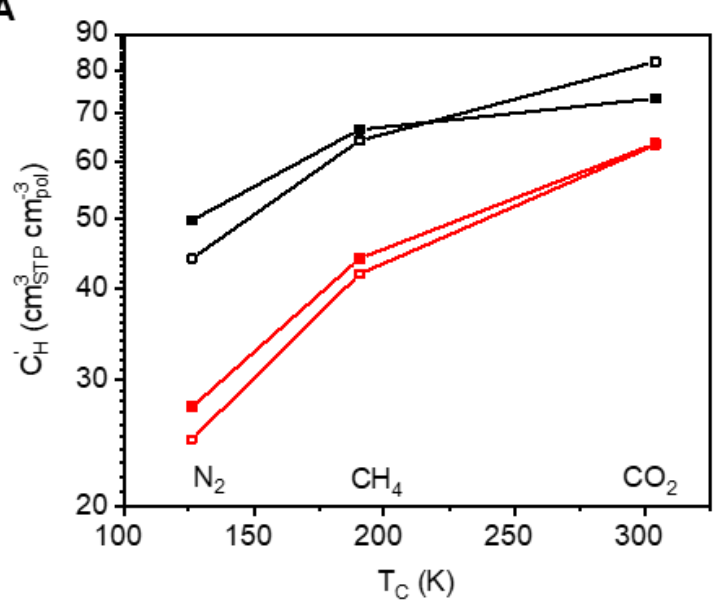

C

C
B

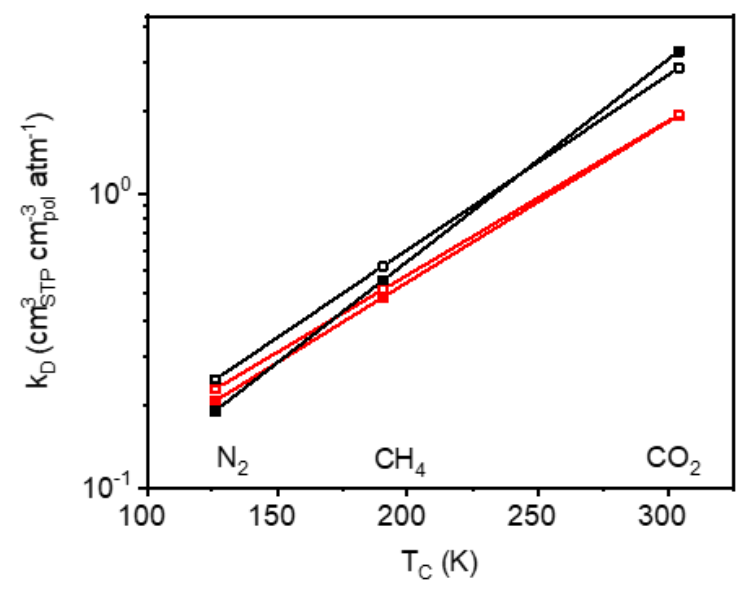

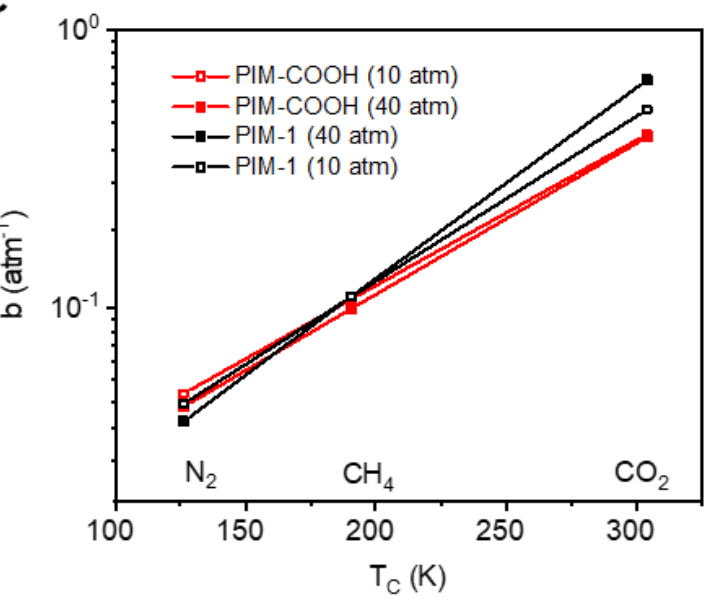

Figure S2. Correlations of (a) $C_{H}^{\prime}$, (b) $k_{D}$ and (c) $b$ with critical temperature. Dual-mode parameters are calculated with the slope of $k_{D}$ and $b$ versus $\mathrm{T}_{\mathrm{c}}$ constrained to be the same as that of the sorption coefficient against $\mathrm{T}_{\mathrm{c}}$ at 10 atm (empty symbols) or 40 atm (filled symbols). This procedure follows a method previously proposed by Smith et al. ${ }^{1}$ 


\section{S6. 2008 Upper-bound performance of PIM-1 and PIM-COOH}
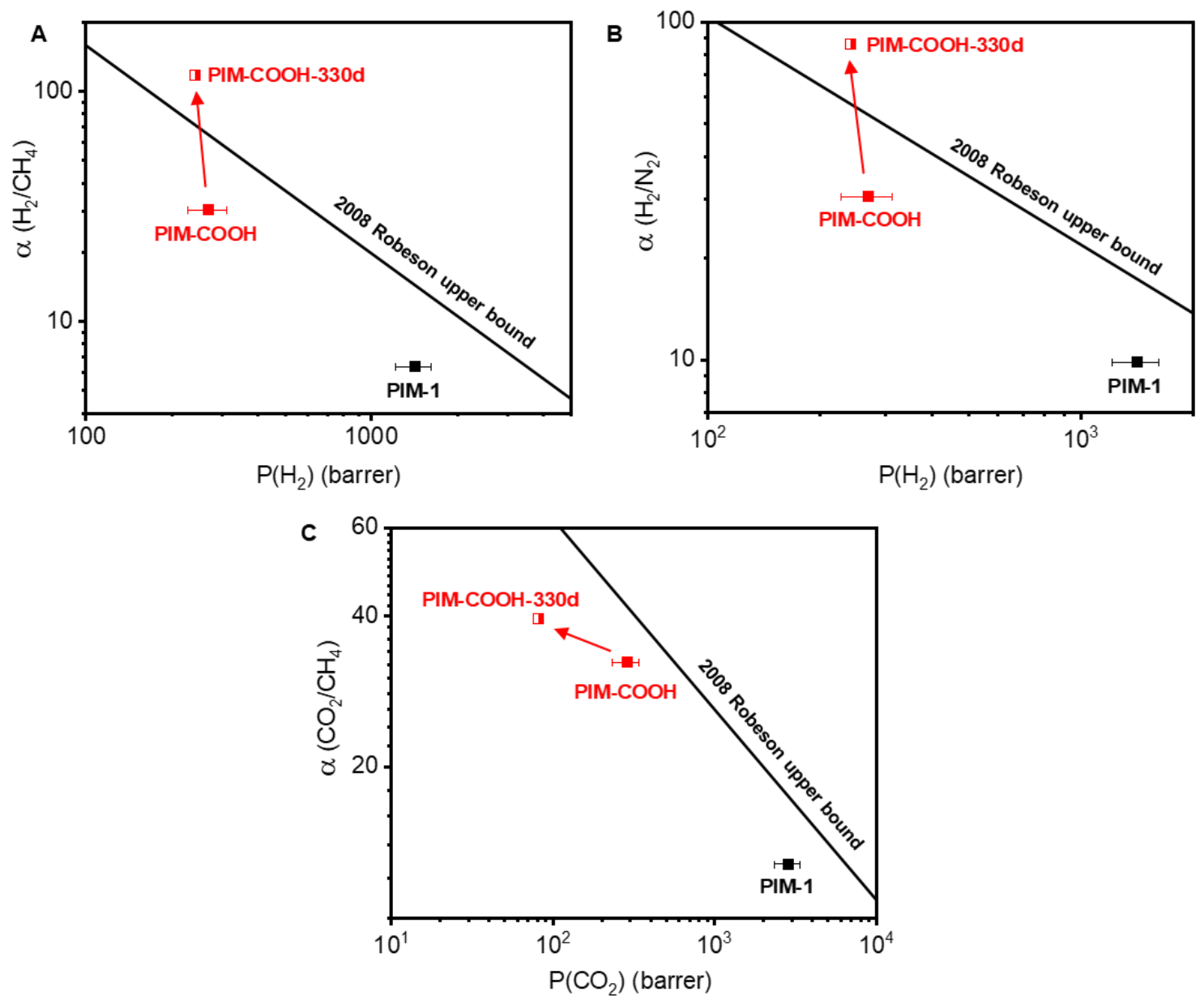

Figure S3. Upper-bound relationship for (a) $\mathrm{H}_{2} / \mathrm{CH}_{4}$, (b) $\mathrm{H}_{2} / \mathrm{N}_{2}$, and (c) $\mathrm{CO}_{2} / \mathrm{CH}_{4}$ gas pairs. Semifilled red squares indicate pure-gas data for a 330 day aged PIM-COOH (PIM-COOH-330d) sample measured at $1 \mathrm{~atm}$ and $35^{\circ} \mathrm{C}$. 


\section{S7. Error Analysis}

Permeability, diffusion coefficients, sorption coefficients, and their respective selectivities calculated from the time-lag method and permeation tests had error reported as standard deviations from at least three independent measurements using films cast independently while treated under the same conditions.

Density measurements were conducted at least five times and errors are reported as standard deviations.

Volume expansions used to calculate the downstream volumes in the pressure-decay sorption systems were performed at least three times at three pressures, and the error associated with these volumes are reported as standard deviations.

The concentration of gas sorbed was calculated from pressure-decay experiments. Error was calculated through a propagation of error analysis ${ }^{2}$. Propagation of error was evaluated for each respective calculation through Equation $\mathrm{S} 1$ :

$\sigma_{f}=\sqrt{\left(\frac{d f}{d x}\right)^{2} \sigma_{x}^{2}+\left(\frac{d f}{d y}\right)^{2} \sigma_{y}^{2}+\left(\frac{d f}{d z}\right)^{2} \sigma_{z}^{2}+\cdots}$

When applicable, propagations of error took into account errors associated with:

The polymer volume calculated from density measurements $\left(\sigma_{p v}\right)$, which also accounted for variation in mass and density values.

The system volumes measured from the helium expansion through the Burnett method ${ }^{3}$.

Valve volume $\left(\sigma_{v v}=0.0002, \mathrm{~cm}^{3}\right)$

Charge volume $\left(\sigma_{v c}=0.01, \mathrm{~cm}^{3}\right)$

Sample volume $\left(\sigma_{v s}=0.009, \mathrm{~cm}^{3}\right)$

The reported accuracy of the temperature controller $\left(\sigma_{T}=0.01, K\right)$.

The reported accuracy of the low-pressure transducer $\left(\sigma_{L P}=0.025\right.$, torr $)$

The reported accuracy of the high-pressure transducer $\left(\sigma_{H P}=0.5, p s i a\right)$ 


\section{References}

(1) Smith, Z. P.; Sanders, D. F.; Ribeiro, C. P.; Guo, R.; Freeman, B. D.; Paul, D. R.; McGrath, J. E.; Swinnea, S. Gas Sorption and Characterization of Thermally Rearranged Polyimides Based on 3,3'-dihydroxy-4,4'-diamino-biphenyl (HAB) and 2,2'-bis-(3,4dicarboxyphenyl) Hexafluoropropane Dianhydride (6FDA). J. Memb. Sci. 2012, 415-416, 558-567.

(2) Bevington, P. R. Data Reduction and Error Analysis for the Physical Sciences McGraw Hill Book Co. New York 1969.

(3) Burnett; E.S. Compressibility Determinations without Volume Measurements. [Apparatus to Determine Compressibility with Pressure and Temperature Measurements], 1936. 